\title{
Nefrolitiasis en la paciente gestante: revisión de la literatura
}

\section{Renal lithiasis in pregnant patient: a literary review}

\author{
José Bohórquez-Rivero*, José Restom-Arrieta1, José Sáenz-López¹, Diego Sánchez-Martínez¹, \\ Mónica Brieva-Deulofeut', Juan P. Rodríguez-Lizarralde² y Emilio Abuabara-Franco ${ }^{3}$ \\ ${ }^{1}$ Grupo de Investigación GIBACUS, Escuela de Medicina, Facultad de Ciencias de la Salud; 'Departamento de Ginecología y Obstetricia; \\ ${ }^{3}$ Departamento de Medicina Interna, Universidad del Sinú Seccional Cartagena. Cartagena de Indias, Colombia
}

\section{Resumen}

La litiasis renal en la paciente gestante es una condición relativamente infrecuente; sin embargo, es la causa más común de dolor no obstétrico durante el embarazo. En el periodo de gestación se producen diversos cambios anatomofisiológicos en el tracto urinario, como la dilatación de los cálices renales, la pelvis renal y los uréteres por causa del efecto que ejerce la progesterona sobre el músculo liso uretral, y la compresión de los uréteres por el útero grávido. Estas modificaciones conducen a un aumento del flujo plasmático renal y de la tasa de filtrado glomerular, ocasionando hiperuricosuria e hipercalciuria. Del mismo modo, durante el embarazo también se produce un aumento de la secreción de inhibidores de cálculos, por lo cual la prevalencia de la formación de cálculos durante el embarazo es similar a la de las mujeres no embarazadas. El bajo índice de sospecha por parte del médico tratante puede entorpecer el diagnóstico y el tratamiento de esta patología, que también son limitados en la gestación debido a los potenciales riesgos teratogénicos. Se realiza una revisión narrativa de la literatura partiendo de la evidencia científica disponible en las diferentes bases de datos y de esta manera se pretende instruir al médico en los aspectos clave de dicho tema.

Palabras clave: Nefrolitiasis. Urolitogénesis. Cálculos. Embarazo. Feto.

\section{Abstract}

Renal lithiasis in pregnant women is a relatively rare condition. However, it is the most common cause of non-obstetric pain during pregnancy. During the gestation period, various anatomical-physiological changes occur in the urinary tract. These changes include dilation of the renal calyces, renal pelvis, and ureters due to the effect of progesterone on urethral smooth muscle and compression of the ureters by the gravid uterus. These modifications lead to an increase in renal plasma flow and glomerular filtration rate, thus causing hyperuricosuria and hypercalciuria. Similarly, during pregnancy there is also an increase in the secretion of stone inhibitors, therefore, the prevalence of stone formation during pregnancy is similar to non-pregnant women. The low index of suspicion on the part of the treating physician can hinder the diagnosis and treatment of this pathology, which is also limited in pregnancy due to teratogenic risks. A narrative review of the literature is carried out based on the scientific evidence available in the different databases and in this way it is intended to instruct the doctor in the key aspects of said topic.

Key words: Nephrolithiasis. Urolithogenesis. Stones. Pregnancy. Fetus. 


\section{Introducción}

La nefrolitiasis es una patología caracterizada por la formación, la agregación y la fijación de cristales en el árbol urinario, con la consecuente aparición de un cálculo $^{1,2}$. Durante el embarazo, la nefrolitiasis es la causa más común de dolor no obstétrico ${ }^{3,4}$.

La nefrolitiasis en la mujer embarazada obedece a una fisiopatología que difiere de la de la mujer no embarazada debido a que durante la gestación se producen ciertas modificaciones anatómicas del árbol urinario, que pueden promover la litogénesis. Del mismo modo, ocurren alteraciones en los parámetros bioquímicos del tracto urinario (aumento de los factores litógenos, pero también de los litoprotectores) y en la dinámica hormonal, que conllevan una nueva homeóstasis, diferente de la que existe sin embarazo ${ }^{4,5}$.

Las posibles complicaciones de la nefrolitiasis pueden poner en peligro la vida tanto de la gestante como la del feto; puede iniciar un trabajo de parto prematuro o interferir con la progresión del trabajo de parto normal, además de los cuadros infecciosos secundarios ${ }^{6}$. Del mismo modo, se ha relacionado con diabetes gestacional, preeclampsia y abortos. Por lo tanto, se hace necesario un diagnóstico rápido y preciso de dicha patología en este grupo poblacional ${ }^{3}$.

La nefrolitiasis en el embarazo plantea dilemas tanto de diagnóstico como de tratamiento. El mayor limitante para el diagnóstico es la exposición del feto a la radiación, debido a que podría ocasionar consecuencias deletéreas?. Además, la nefrolitiasis coincide con la presentación clínica de otras patologías agudas, como apendicitis, diverticulitis o desprendimiento prematuro de placenta, lo que retrasa aún más el diagnóstico ${ }^{6}$. El tratamiento de esta afección a menudo requiere un abordaje multidisciplinario que implica la participación simultánea del urólogo, el obstetra, el neonatólogo y el anestesiólogo. Los efectos adversos del uso de anestesia, fármacos y cirugía en la madre y el feto limitan la utilización del arsenal completo de modalidades terapéuticas que se usan comúnmente en las mujeres no embarazadas ${ }^{6}$.

\section{Epidemiología}

La incidencia de cálculos en las mujeres gestantes es idéntica a la de las mujeres no gestantes ${ }^{8}$, y es de 1 por cada 1500 embarazos $^{9,10}$. Los cálculos en las mujeres suelen manifestarse entre la tercera y la quinta décadas de la vida, con una edad promedio de 24,6 $a_{n ̃ o s}{ }^{6}$. La gran mayoría de los cálculos en el embarazo se descubren en el segundo o tercer trimestre; numerosos estudios reportan una distribución de los cálculos de aproximadamente el $20 \%$ en el primer trimestre, el $40 \%$ en el segundo y el $46 \%$ en el tercero ${ }^{10}$. Se presenta con mayor frecuencia en mujeres multíparas que en nulíparas ${ }^{6,9}$. Se ha reportado que la incidencia de nefrolitiasis sintomática que complica el embarazo es de 1 por cada 3300 embarazos. Ambos riñones y ambos uréteres pueden verse afectados por igual. Sin embargo, en el momento de la presentación, los cálculos se localizan con mayor frecuencia en el uréter que en la pelvis renal y los cálices renales. Se ha registrado la recurrencia de cálculos renales en el $25 \%$ de las pacientes embarazadas ${ }^{6}$.

\section{Factores de riesgo}

Los factores de riesgo son similares a los de la población general. Dentro de ellos se encuentran la edad (tercera a quinta décadas de la vida), los antecedentes familiares de nefrolitiasis, los cálculos de oxalato de calcio o de fosfato de calcio (hidroxiapatita) y tener un solo riñón funcional (monorreno) $)^{9,11,12}$. Del mismo modo, los factores geográficos (las mujeres blancas están más predispuestas al desarrollo de cálculos urinarios que las hispanas, negras y asiáticas), climáticos (clima cálido y seco) y dietéticos (baja ingesta de fluidos, alto consumo de calcio, sodio, fructosa y carnes rojas), así como el estilo de vida (sedentarismo, obesidad), también influyen en el desarrollo de cálculos renales en este grupo poblacional, lo que lleva a una variación epidemiológica dependiente del entorno en que se encuentre la paciente $6,13,14$.

Entre otros factores que contribuyen a la aparición de cálculos renales se encuentran algunas enfermedades sistémicas, principalmente la diabetes mellitus y la hipertensión arterial. La diabetes mellitus se considera un factor independiente para el desarrollo de nefrolitiasis primaria y recurrente ${ }^{6}$. Otras patologías que incrementan el riesgo de nefrolitiasis son el hiperparatiroidismo, la acidosis tubular renal, la cistinuria, la hiperoxaluria primaria, los antecedentes de derivación yeyunoileal, la enfermedad de Crohn, los antecedentes de resección intestinal, el síndrome de hipoabsorción, la sarcoidosis y el hipertiroidismo ${ }^{11}$. La ingesta de algunos suplementos o fármacos también se ha relacionado con la aparición de cálculos; por ejemplo, suplementos de calcio, vitamina $D$, megadosis de ácido ascórbico (> $4 \mathrm{~g}$ al día) o fármacos como la acetazolamida, las sulfonamidas, el triamtereno, el indinavir y el aciclovir, entre otros ${ }^{6,10}$. 
Es importante mencionar que muchas mujeres embarazadas pueden estar tomando suplementos de calcio adicionales según la evidencia de que reduce significativamente el riesgo de preeclampsia, morbimortalidad materna y parto prematuro ${ }^{15}$. A pesar de que los investigadores han encontrado una tendencia hacia un mayor riesgo de nefrolitiasis con la suplementación de calcio durante el embarazo, no ha sido estadísticamente significativa ${ }^{16}$. Sin embargo, los beneficios de la suplementación de calcio durante el embarazo se deben sopesar cuidadosamente con los riesgos potenciales de nefrolitiasis, en especial en gestantes con alto riesgo de padecerla ${ }^{17}$. De igual manera, los antecedentes de cirugía de bypass gástrico y cirugía bariátrica, entre otras, se han relacionado con un aumento en el riesgo de aparición de nefrolitiasis, al igual que en la población general ${ }^{13,14,18}$.

También existen factores propios de cada paciente que predisponen a la aparición de cálculos, como bajo volumen urinario, alta concentración de la orina, alteraciones del $\mathrm{pH}$, alojamiento de cuerpos extraños (material de sutura, catéter, sondas de Foley, dispositivos intrauterinos), obstrucción de la salida vesical que ocasiona estasis urinaria, condensación del sedimento urinario y cristalización, prolapso de los órganos pélvicos o divertículo uretral, entre otros ${ }^{15,18}$. Las alteraciones anatómicas relacionadas con la formación de cálculos y que deben estudiarse son la ectasia tubular renal o riñón en esponja, el riñón en herradura, los divertículos y quistes en los cálices renales, la obstrucción de la unión pieloureteral, la estenosis ureteral, el reflujo vesicoureteral y el ureterocele ${ }^{10}$.

\section{Etiología de los cálculos durante la gestación}

\section{Cambios anatomofisiológicos renales}

Los cambios anatomofisiológicos en el sistema renal constituyen una parte sustancial en el desarrollo de la nefrolitiasis en la paciente gestante ${ }^{3,19}$. Estas modificaciones homeostáticas conducen en ciertas gestantes a la génesis de cálculos renales, y con ello pueden dimanar otras complicaciones ${ }^{4,20}$.

Entre las modificaciones del tracto urinario durante el embarazo se encuentra la hidroureteronefrosis. Se han planteado dos mecanismos para explicar la posible causa de la dilatación del sistema colector urinario en el embarazo: la teoría hormonal y la teoría mecánica. La teoría hormonal postula que es producto de la hipotonía en las vías excretoras, secundaria a la relajación del músculo liso y a la disminución del peristaltismo ureteral mediado por el aumento de la progesterona y las prostaglandinas. Por otro lado, la teoría mecánica postula que es producto de la compresión de los uréteres por el útero grávido y los vasos iliacos y gonadales. Dicha dilatación fisiológica se produce en el $90 \%$ de las embarazadas, ocurre aproximadamente entre las semanas 6 y 10 de gestación, incrementa en todo el periodo gestacional y desaparece en 4 a 6 semanas del puerperio ${ }^{10,11,21,22}$. Típicamente, la hidroureteronefrosis exhibe un predominio mayor en el lado derecho, que puede deberse a la dextrorrotación uterina y al papel protector que brinda el colon sigmoides al uréter izquierdo por su contenido gaseoso ${ }^{18,21}$. La hidroureteronefrosis produce estasis urinaria y aumenta el tiempo de contacto con factores litogénicos urinarios, lo que puede incrementar el potencial de cristalización y la formación de cálculos. Del mismo modo, la dilatación del tracto urinario facilita la migración de cálculos renales asintomáticos desde el riñón al uréter, lo que podría explicar la observación de que los cálculos ureterales son dos veces más comunes que los cálculos renales. En la mayoría de los casos, la hidroureteronefrosis gestacional es asintomática y no se asocia con obstrucción significativa; sin embargo, en ocasiones puede resultar en dolor en el flanco e incluso en rotura del fórnix ${ }^{10,22}$.

De igual forma, durante el primer trimestre de embarazo se produce un aumento significativo del gasto cardiaco y del volumen sanguíneo circulante, aunado a una disminución de la resistencia vascular periférica, lo que ocasiona un incremento del flujo plasmático renal, con la consecuente elevación de la tasa de filtración glomerular, que puede ser superior al 50\% $\%$ 17,19,23. Esto da como resultado un aumento en la excreción urinaria de constituyentes litogénicos, incluidos calcio, ácido úrico, oxalato y sodio; este hecho constituye un factor de riesgo para la aparición de cálculos ${ }^{17,24}$.

La hipercalciuria se intensifica gracias a la síntesis de 1,25-dihidroxi-colecalciferol (1-25-hidroxi-vitamina D) por la placenta, lo cual produce un aumento de la absorción intestinal de calcio con el fin de garantizar al feto un adecuado aporte de este elemento y permitir la formación del esqueleto fetal; también ocasiona un descenso fisiológico de la hormona paratiroidea, cuyo hipoparatiroidismo fisiológico causa una disminución de la reabsorción tubular del calcio y un aumento de su excreción urinaria ${ }^{6,10,11}$.

El aumento de los factores litogénicos durante el embarazo se equilibra con un aumento similar en la excreción de inhibidores de cálculos urinarios ${ }^{17}$; es decir, 
al mismo tiempo, existen mecanismos que ayudan a regular y contrarrestar la aparición de cálculos renales ${ }^{11}$. El aumento de la tasa de filtración glomerular causa la excreción de citrato y de magnesio, que cumplen una acción litoprotectora; dichos iones se unen al calcio y al oxalato, respectivamente, haciéndolos más solubles. El citrato, al unirse a los iones de calcio, disminuye la cantidad circulante de calcio y por tanto su capacidad de unirse al fosfato y al oxalato; y el magnesio, al unirse al oxalato, reduce la unión de este al calcio. Estos hechos perturban la génesis de cristales de fosfato cálcico y oxalato, los cuales predisponen a la aparición de nefrolitiasis. Del mismo modo, aumenta la excreción de glucosaminoglicanos, nefrocalcina, uromodulina y tiosulfato, los cuales también inhiben el crecimiento y la agregación de cristales. Este mecanismo es igual en las mujeres no embarazadas, y por lo tanto la incidencia de la nefrolitiasis es igual en las embarazadas que en las no embarazadas, aunque la composición de los cálculos en las gestantes suele ser diferente de la que se observa fuera del embarazo $6,10,11,17$.

\section{Mecanismo de formación de cálculos renales}

El proceso de litogénesis es una cascada compleja de eventos que requiere una orina sobresaturada de sales formadoras de cálculos, la falta de inhibidores de la formación de cálculos (citrato y glucoproteínas) y la retención de cristales o núcleos en el riñón en sitios de anclaje que promueven la agregación y el crecimiento del cálculo ${ }^{11}$ (Fig. 1).

La composición de los cristales depende de los mecanismos implicados en su formación; pueden ser cristales de ácido úrico (disminución del pH, disminución del volumen urinario, aumento de ácido úrico), de cistina (aumento de la cistina) o secundarios a infecciones $(\mathrm{pH}$ elevado, iones de amonio, sustancias mucosas), entre otros ${ }^{18,25}$. Esto culmina en un incremento vasto de la saturación de la orina, provocando la formación del cálculo ${ }^{26,27}$.

Durante el embarazo, el aumento del citrato en la orina eleva el pH urinario y esta condición contribuye a la prevención de la formación de cristales de oxalato de calcio y ácido úrico; sin embargo, la alcalinización del $\mathrm{pH}$ urinario se asocia a la formación de cristales de fosfato cálcico (disminución del volumen, aumento del calcio y aumento del oxalato, aunado a disminución de las sustancias con propiedades inhibitorias de la formación de cálculos, como los inhibidores macromoleculares), que son predominantes en la gestante ${ }^{17,28}$.
En el embarazo, por la razón previamente expuesta, la constitución de los cálculos es en su mayoría de fosfato de calcio (hidroxiapatita), en un $66,6 \%$, seguidos por los cálculos de oxalato de calcio, que son mucho menos frecuentes ${ }^{8,10,26,29}$. Los cálculos de ácido úrico, cistina y estruvita (fosfato de amonio y magnesio hexahidratado, cálculos de infección) se encuentran con menos frecuencia ${ }^{6,7}$. Las variantes raras de los cálculos incluyen cálculos de fármacos y de urato de ácido amónico $(1 \%)^{13}$.

\section{Manifestaciones clínicas}

Los cálculos renales pueden ser asintomáticos 0 manifestarse con una amplia gama de signos y síntomas $^{18}$. La presentación clínica se amolda a las características individuales de cada caso y a las propiedades del cálculo en función de la fisiopatología.

La nefrolitiasis se manifiesta como un dolor de tipo cólico, de inicio impetuoso, creciente y discontinuo, con una duración episódica de 20-60 minutos, localizado en el flanco (usualmente unilateral) y con predominio nocturno ${ }^{13,30}$. Tanto la localización del dolor como la intensidad obedecen a la posición del cálculo y sus dimensiones ${ }^{30,31}$. La hematuria está presente en gran número de pacientes; sin embargo, un mayor lapso de tiempo entre la primera sensación de dolor y la prueba de orina incrementa la probabilidad de que la hematuria no se evidencie ${ }^{14}$. En un estudio retrospectivo de 452 pacientes realizado por Kobayashi, et al. ${ }^{32}$, la hematuria estuvo presente en el $95 \%$ de los pacientes en que la prueba se realizó el primer día, y dicho porcentaje disminuyó al $65 \%$ al tercer día.

A medida que el cálculo desciende, la situación del dolor cambia y se asocia a polaquiuria y disuria ${ }^{13}$. La cápsula renal y los intestinos comparten la inervación esplácnica del plexo celíaco, y por ello los pacientes también pueden presentar náuseas y vómitos ${ }^{13,30}$. La presencia de fiebre se asocia a infección. Los síntomas como la taquicardia y la hipertensión generalmente son secundarios al dolor ${ }^{13,31}$.

En las pacientes gestantes, las manifestaciones son similares; sin embargo, la sospecha de nefrolitiasis en este grupo poblacional representa un desafío para el clínico ${ }^{17}$. A pesar de que la nefrolitiasis es la causa no obstétrica más común de dolor renal, hay una desmesurada lista de causas obstétricas que pueden mimetizar un cólico renal, lo que distrae el juicio del galeno y obstaculiza el diagnóstico oportuno. Por ejemplo, la elevación de la prolactina en la gestante puede provocar náuseas y vómitos con mayor frecuencia durante 


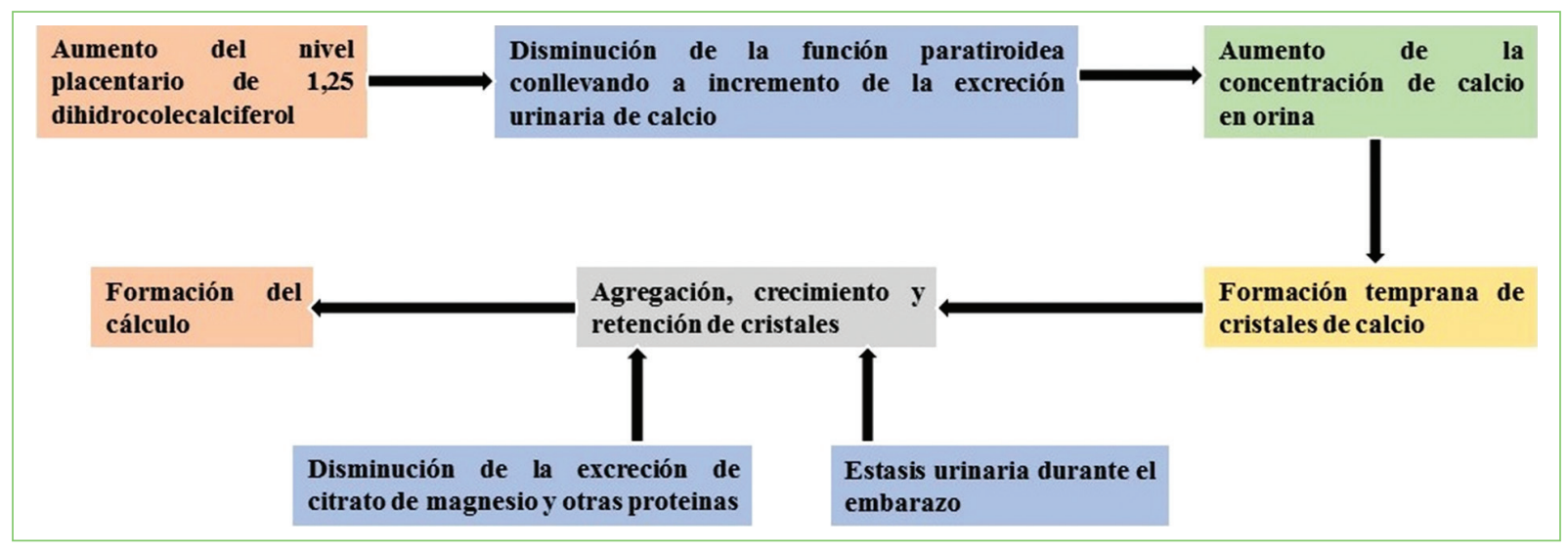

Figura 1. Mecanismo de la litogénesis durante el embarazo. (Adaptada de Martínez ${ }^{11}$.)

el primer trimestre, pero pueden estar presentes durante todo el embarazo; un alto porcentaje de pacientes refieren dolor de espalda y abdominal secundario a la lordosis lumbar fisiológica del embarazo y al crecimiento uterino y el estiramiento de los ligamentos redondos y de la musculatura, respectivamente; y la rotura de pequeñas venas de la pirámide renal resultan en hematuria no patológica del embarazo, entre otras $4,8,17,20$. Se conoce que hasta un $30 \%$ de los casos son mal diagnosticados, por lo que es imprescindible un alto índice de sospecha para asegurar un diagnóstico rápido y preciso de nefrolitiasis en la paciente gestante ${ }^{33}$.

Numerosos estudios han demostrado que la nefrolitiasis durante el embarazo podría ocasionar complicaciones tanto maternas como fetales, tales como trabajo de parto pretérmino, parto pretérmino, ruptura prematura de membranas, preeclampsia, diabetes gestacional, endometritis, infecciones del tracto urinario, pérdidas recurrentes del embarazo y síndrome de abstinencia neonatal ${ }^{4,8,9,34}$.

\section{Pruebas diagnósticas}

Los estudios imagenológicos son la piedra angular para la investigación de un cólico renal en el embarazo debido a la dificultad de hacer un diagnóstico preciso basado únicamente en la anamnesis y la exploración física ${ }^{17}$. Se han utilizado diversas modalidades de imagen: ecografía renal, resonancia magnética (RM), tomografía computarizada (TC), radiografía simple y urografía intravenosa ${ }^{31,35,36}$. La necesidad de un diagnóstico preciso y oportuno se debe sopesar cuidadosamente con los riesgos potenciales de exposición a la radiación para la madre y especialmente para el feto ${ }^{17}$. Para lo anterior, se debe partir del principio ALARA (as low as reasonably achievable). El riesgo de teratogenicidad por la exposición del feto a la radiación depende de la edad gestacional en el momento de la exposición. El umbral estimado para inducir teratogénesis o aborto espontáneo en el primer trimestre es de 20 mGy, y de 50 mGy en el segundo y el tercer trimestre $^{37}$. Sin embargo, se cree que la radiación tiene un efecto estocástico sobre la carcinogénesis, y que no existe un «umbral seguro» absoluto de exposición; por lo tanto, siempre se debe informar de los riesgos incluso cuando la exposición se considere leve ${ }^{38}$. El riesgo de cáncer infantil secundario a la exposición en el útero a 10 mGy de radiación se estima que es de 1 en 10,000 ${ }^{17}$. El American College of Obstetricians and Gynecologists (ACOG) opina que las dosis de radiación inferiores a 50 mGy durante los embarazos son seguras para el feto, sin un mayor riesgo de pérdida del embarazo ni de anomalías fetales ${ }^{38}$. Las dosis fetales aproximadas de radiación para las modalidades de imagen habituales se detallan en la tabla 1.

La ecografía renal constituye la herramienta diagnóstica de primera línea ante la sospecha de cálculos en la paciente gestante. Tiene numerosos beneficios, pues brinda seguridad tanto para la madre como para el feto debido a la no exposición a radiación ionizante, es rápida y resulta fácilmente accesible en los centros de salud. La ecografía muestra el parénquima renal y el sistema pielocalicial, el uréter superior y parte del uréter inferior a través de la vejiga ${ }^{19}$; sin embargo, un diagnóstico definitivo con ecografía puede ser difícil debido al hábito corporal de la paciente, la posición del feto o la ubicación de los cálculos dentro del uréter por 
Tabla 1. Cantidad de radiación que pasa al feto según la técnica utilizada y el sitio anatómico

\begin{tabular}{|l|l|c|}
\hline $\begin{array}{l}\text { Categoría de } \\
\text { exposición }\end{array}$ & Sitio de exploración & $\begin{array}{c}\text { Dosis de radiación } \\
\text { fetal (mGy) }\end{array}$ \\
\hline $\begin{array}{l}\text { Dosis muy bajas } \\
<<0,1 \mathrm{mGy})\end{array}$ & $\begin{array}{l}\text { Columna cervical } \\
\text { (anteroposterior y } \\
\text { lateral) }\end{array}$ & $<0,001$ \\
\hline & Cualquier extremidad & 0,001 \\
\hline $\begin{array}{l}\text { Dosis bajas a } \\
\text { moderadas } \\
\text { (0,1-10 mGy) }\end{array}$ & Tórax (dos vistas) & $0,0005-0,01$ \\
\hline $\begin{array}{l}\text { Columna lumbar } \\
\text { Tosis muy bajas } \\
\text { <0,1 mGy) }\end{array}$ & Cabeza o cuello & $0,1-3$ \\
\hline $\begin{array}{l}\text { Dosis bajas a } \\
\text { moderadas } \\
\text { (0,1-10 mGy) }\end{array}$ & $\begin{array}{l}\text { Tórax o angiografía } \\
\text { pulmonar }\end{array}$ & $1-10$ \\
\hline $\begin{array}{l}\text { Dosis altas } \\
\text { Abdominal }\end{array}$ & Pelvis & $0,01-0,66$ \\
\hline
\end{tabular}

Adaptada de American College of Obstetricians and Gynecologists ${ }^{38}$.

el útero grávido ${ }^{17,35,39}$. La ecografía transvaginal puede aportar información valiosa, ya que evalúa mejor el uréter distal y la unión ureterovesical. Se ha reportado una sensibilidad del $34 \%$ y una especificidad del $86 \%$ para la ecografía en la detección de nefrolitiasis en el embarazo $^{\circ}$. La baja sensibilidad se debe a que suele ser difícil distinguir entre obstrucción ureteral secundaria a cálculos e hidroureteronefrosis fisiológica por compresión mecánica del uréter entre el útero grávido y el músculo psoas ilíaco. La ecografía no puede visualizar una sección larga del uréter en el retroperitoneo 9,17 . En un intento por mejorar la precisión diagnóstica de la ecografía, se han utilizado diversas medidas complementarias que incluyen chorros ureterales e índices de resistencia $(\mathrm{IR})^{17}$.

La ecografía Doppler color se puede utilizar para visualizar el artefacto de centelleo del cálculo y para detectar el paso de orina en la unión ureterovesical. El chorro ureteral, producido por el paso de orina desde el extremo distal de cada uréter hasta a la vejiga, si existe indica que no es una oclusión completa; debe evaluarse durante al menos 5 minutos, y un chorro ureteral ausente, asimétrico o reducido en el lado sintomático es particularmente sensible $(95 \%)$ y específico $(87 \%)$ para la obstrucción en pacientes no embarazadas, pero no tanto en las embarazadas, ya que el chorro ureteral puede estar ausente en el 13-15\% de las mujeres gestantes asintomáticas ${ }^{9,17}$. La obtención de imágenes en una posición de decúbito lateral puede disminuir los falsos positivos. La hidratación de la paciente ayuda a mejorar la precisión del diagnóstico, al dilatar la vejiga y optimizar la determinación del chorro ureteral. También se ha implementado la ecografía Doppler con la medición del IR para distinguir la hidroureteronefrosis fisiológica de la obstrucción patológica. Se ha sugerido un IR $>0,70$ o más como marcador de obstrucción ${ }^{9,10,11}$. Sin embargo, el IR es inespecífico y puede estar elevado en riñones normales, y también puede no aumentar durante las primeras fases de la obstrucción, cuando hay dilatación vascular ${ }^{17}$.

La RM debe ser considerada una prueba de segunda línea cuando la ecografía falla y con el manejo conservador los síntomas aún persisten. Este estudio tiene una sensibilidad del $77 \%$ y una especificidad del $83 \% 7,40$. También se ha utilizado la urografía por RM (uro-RM) sin contraste (protocolo HASTE [Half Fourier Acquisition Single Shot Turbo Spin Echo]) en pacientes embarazadas con nefrolitiasis, con mayor precisión que la ecografía ${ }^{6}$. El protocolo HASTE ha surgido como una opción prometedora para el diagnóstico por imagen de la nefrolitiasis en el embarazo. Dicho protocolo evita las radiaciones ionizantes, no se conocen efectos nocivos para el feto, tiene un tiempo de adquisición rápido (unos 15 minutos) y puede evaluar las causas de los síntomas no urológicos ${ }^{41}$. Sin embargo, su uso está limitado por el costo, la disponibilidad y la imposibilidad de ser utilizado en pacientes con implantes metálicos ${ }^{17}$. Los hallazgos característicos de la obstrucción patológica incluyen la visualización directa de un cálculo en un punto de constricción ureteral, edema renal o extravasación perirrenal, y el signo de «doble torcedura" (la constricción está presente en el borde pélvico y la unión ureterovesical ${ }^{41}$. Una pequeña serie ha demostrado que la uro-RM con protocolo HASTE tiene una sensibilidad del $84 \%$, una especificidad del $100 \%$ y una precisión diagnóstica del $100 \%$ en la obstrucción ureteral aguda durante el embarazo ${ }^{42}$.

La TC tiene una sensibilidad del $97 \%$ y una especificidad del $96 \%{ }^{18}$ (Tabla 2). Su ventaja radica en que brinda la localización y la medición precisas del cálculo dentro del árbol urinario, y además es útil para el diagnóstico diferencial de patologías que cursan con la misma sintomatología ${ }^{18,31}$. No obstante, su mayor desventaja para la paciente gestante es la alta exposición del feto a la radiación; de hecho, este es el factor 
Tabla 2. Pruebas diagnósticas para nefrolitiasis en embarazadas

\begin{tabular}{|c|c|c|c|}
\hline Pruebas diagnósticas & Confiabilidad & Ventajas & Desventajas \\
\hline Ecografía renal & $\begin{array}{l}\text { Sensibilidad: } 34 \% \\
\text { Especificidad: } \\
86 \%^{9}\end{array}$ & $\begin{array}{l}\text { Menor exposición del feto a la radiación } \\
\text { Menor costo } \\
\text { Mayor disponibilidad }^{3}\end{array}$ & $\begin{array}{l}\text { Inespecífica al diferenciar } \\
\text { hidroureteronefrosis fisiológica del } \\
\text { embarazo frente a una obstrucción } \\
\text { causada por cálculos renales } \\
\text { Limitación por la presencia de gas } \\
\text { intestinal } \\
\text { Limitación según el biotipo } \\
\text { morfológico de la paciente }\end{array}$ \\
\hline $\begin{array}{l}\text { Resonancia } \\
\text { magnética }\end{array}$ & $\begin{array}{l}\text { Sensibilidad: } 77 \% \\
\text { Especificidad: } \\
83 \% 18,31\end{array}$ & $\begin{array}{l}\text { Proporciona imágenes de alta calidad de los } \\
\text { riñones y el tracto urinario sin radiación } \\
\text { ionizante } \\
\text { Permite la visualización de signos secundarios } \\
\text { de obstrucción (agrandamiento renal asimétrico, } \\
\text { edema renal o perirrenal, edema periureteral, } \\
\text { entre otros) o defectos de llenamiento } \\
\text { Distingue la dilatación fisiológica del uréter de } \\
\text { una uropatía obstructiva subyacente }\end{array}$ & $\begin{array}{l}\text { No es costo-efectiva y no está } \\
\text { disponible en todos los centros de } \\
\text { salud }^{3}\end{array}$ \\
\hline $\begin{array}{l}\text { Tomografía } \\
\text { computarizada }\end{array}$ & $\begin{array}{l}\text { Sensibilidad: } 97 \% \\
\text { Especificidad: } \\
96 \%{ }^{18}\end{array}$ & $\begin{array}{l}\text { Localización y medición precisa del cálculo } \\
\text { dentro del tracto urinario } \\
\text { Útil en el diagnóstico diferencial de otras } \\
\text { patologías }{ }^{18,31}\end{array}$ & $\begin{array}{l}\text { Alta exposición del feto a la } \\
\text { radiación }\end{array}$ \\
\hline
\end{tabular}

limitante más importante ${ }^{3,39}$. Como ya se ha mencionado, se ha demostrado que dosis de radiación menores de 50 mGy se asocian a un riesgo más bajo de malformaciones congénitas, retraso en el crecimiento intrauterino, efectos carcinogénicos y muerte fetal intrauterina ${ }^{38}$. En este sentido, el uso sensato de protocolos de TC de dosis baja, es decir, que exponen al feto a dosis más bajas de radiación y mantienen la precisión diagnóstica, puede ser una opción valiosa ${ }^{6,7,36,43}$. Una serie que examinó la TC de dosis baja durante el embarazo confirmó una menor exposición a la radiación, de aproximadamente 7,1 mGy ${ }^{44}$. Además, un estudio multiinstitucional demostró que la TC en dosis bajas tenía un valor predictivo positivo más alto $(96 \%)$ que otras modalidades de imagen para detectar nefrolitiasis en el embarazo ${ }^{45}$. La American Urological Association recomienda la TC de dosis baja (definida como $<5 \mathrm{mGy}$ ) como una modalidad de imagen adecuada para mujeres en el segundo o tercer trimestre de embarazo cuando la ecografía inicial no es diagnóstica, basándose en el respaldo del ACOG de que esta está muy por debajo del umbral de radiación de 50 mGy y no se asocia con pérdidas ni anomalías fetales ${ }^{38,46}$.

La urografía intravenosa y la radiografía simple de abdomen se encuentran en desuso debido a los avances tecnológicos en las pruebas de imagen como la ecografía, la RM y la TC de bajas dosis; sin embargo, pueden desempeñar un papel complementario en el diagnóstico de cálculos renales en esta población en particular cuando los hallazgos ecográficos no son concluyentes ${ }^{6,9,18,47}$.

Se indican otras pruebas de laboratorio complementarias para el diagnóstico de litiasis renal, como hemograma, pruebas de función renal, pruebas de coagulación, uroanálisis y urocultivo ante la presencia de fiebre $o$ de nitritos $30,47-49$.

\section{Tratamiento}

El tratamiento de la nefrolitiasis en la embarazada también representa un desafío para el médico y usualmente es necesaria la intervención simultánea de un equipo multidisciplinario, dadas las posibles complicaciones que podrían poner en peligro el bienestar de la paciente y del feto ${ }^{24}$. El equipo debe estar conformado por urólogos, obstetras, neonatólogos y anestesiólo$\operatorname{gos}^{48,49,50}$. Su objetivo es la eliminación de los cálculos con los medios menos invasivos posible para evitar complicaciones como la pérdida de la función renal ${ }^{26,51}$; esto representa un reto debido a que no siempre se cuenta con la ubicación precisa del cálculo.

\section{Manejo conservador}

El abordaje terapéutico en las pacientes embarazadas con nefrolitiasis inicialmente consiste en un manejo conservador. La atención inicial debe proporcionar un 
control adecuado del dolor y evitar la deshidratación. Las pacientes con un cólico renal confirmado durante el embarazo deben iniciar un régimen de analgésicos, hidratación, antieméticos (si están indicados) y antibióticos (si se documenta infección) 6

Una hidratación adecuada debería incrementar el flujo de orina a través del riñón y el uréter, aumentando así la posibilidad de que los cálculos se expulsen espontáneamente. Se prefiere la hidratación oral, a menos de que estén asociados cuadros eméticos ${ }^{6}$.

En la mayoría de los casos basta con la administración de analgésicos para aliviar el dolor y esperar la expulsión del cálculo, que ocurre en el $70 \%$ de las pacientes $^{4,10}$. El manejo del dolor estará guiado por la intensidad de la presentación clínica. Inicialmente deben administrarse analgésicos orales, dentro de los cuales, el de primera línea es el acetaminofén en dosis habituales, por ser uno de los medicamentos más seguros; es decir, no se asocia a malformaciones fetales y puede utilizarse durante todo el embarazo ${ }^{10,52}$. Los antiinflamatorios no esteroideos no se recomiendan debido a las consecuencias fetales adversas (su uso en el tercer trimestre se asocia con oligohidramnios y cierre prematuro del conducto arterioso fetal) ${ }^{10,53,54}$.

Si los analgésicos de primera línea no son eficaces se da inicio a los de segunda línea, dentro de los cuales, los opiáceos pasan a ser los protagonistas, entre ellos la oxicodona, la hidrocodona, la morfina y el fentanilo, los cuales se pueden administrar a lo largo de todo el embarazo; sin embargo, se debe tener precaución ya que el uso prolongado de estos medicamentos puede causar síndrome de abstinencia neonatal ${ }^{54}$. En las pacientes pacientes que no toleran la vía oral (debido a los vómitos) o que no responden a los medicamentos orales se pueden usar analgésicos intravenosos ${ }^{6}$.

Con poca frecuencia, las pacientes pueden seguir experimentando dolor intenso o resistente al tratamiento, y puede ser necesaria una infusión continua epidural de narcóticos ${ }^{6}$. El uso de la terapia médico-expulsiva con alfabloqueantes es una práctica controvertida durante el embarazo, por los riesgos que pueden comportar para la madre y el feto ${ }^{55}$. No obstante, Theriault, et al. ${ }^{55}$, en su estudio sobre el uso de tamsulosina en pacientes embarazadas, demostraron que la administración de este fármaco a corto plazo no se asoció a desenlaces negativos en el feto ni en la madre, y por tanto podría utilizarse como alternativa en el tratamiento conservador, pero es necesario consultar con el obstetra tratante antes de su administración ${ }^{54}$. El mecanismo de acción de estos medicamentos consiste en acelerar la expulsión del cálculo reduciendo el espasmo del músculo liso ureteral ${ }^{56}$.

Como ya se mencionó, los antibióticos en el manejo conservador están indicados en casos específicos, como en el contexto de dolor lumbar, febril o no, con estudio citobacteriológico de orina (ECBO) positivo o no contributivo, pero con claro síndrome de respuesta inflamatoria sistémica (SIRS), sin otro signo de alarma; cálculo urinario probado con ECBO positivo o SIRS ${ }^{10}$. Las penicilinas, las cefalosporinas, la azitromicina, la eritromicina y la clindamicina son antibióticos que pueden ser usados durante el embarazo, considerados como seguros. Los aminoglucósidos no se aconsejan por el riesgo de nefrotoxicidad fetal y materna. Otros medicamentos, como la doxiciclina, las fluoroquinolonas y la trimetoprima, se deben evitar en la paciente embarazada por la evidencia no favorable registrada en estudios con animales. Por otra parte, las sulfonamidas, aunque no han demostrado un riesgo significativo, se deben evitar en fechas cercanas al parto, puesto que pueden generar hiperbilirrubinemia en el recién nacido ${ }^{57}$.

Por último, otras medidas no farmacológicas que pueden ser beneficiosas en el tratamiento conservador son el reposo y la dieta. Se aconseja acostarse en decúbito lateral con el lado sintomático hacia arriba con el fin de aliviar la presión en el uréter ocasionada por el útero grávido ${ }^{48,58}$.

Los enfoques dirigidos a la composición de los cálculos a menudo deben personalizarse durante el embarazo. El enfoque médico más seguro para la litiasis cálcica durante el embarazo es aumentar la ingesta de líquidos y evitar la ingesta excesiva de calcio (incluidas las vitaminas prenatales fortificadas con calcio), sodio y proteínas. La ingesta diaria de calcio no debe exceder los 1000-1200 mg. En las pacientes con antecedentes de cálculos de oxalato de calcio es recomendable el uso de té de hierbas para reducir la excreción urinaria de oxalato, y limitar el consumo de alimentos ricos en oxalato, como espinacas, chocolate y ruibarbo ${ }^{48,58}$. Los diuréticos tiazídicos reducen notablemente la calciuria y la formación resultante de cálculos. Sin embargo, están contraindicados durante el embarazo debido a sus efectos adversos maternos (interfieren con la expansión normal del volumen extracelular del embarazo) y fetales (trombocitopenia, hipoglucemia e hiponatremia).

Los pilares para la prevención de la nefrolitiasis por ácido úrico en el embarazo son una mayor ingesta de líquidos, una ingesta dietética restringida de purinas y la alcalinización urinaria. Los inhibidores de la xantina 
oxidasa, como el alopurinol, que previenen la formación de cálculos de ácido úrico al inhibir la vía final de síntesis de purinas, están contraindicados durante el embarazo debido a los posibles efectos adversos en el feto ${ }^{6}$.

El manejo médico cuidadoso puede permitir que las mujeres con cistinuria y litiasis por cisteína sigan su embarazo de manera segura sin un mayor riesgo. El aumento del volumen urinario a $3000 \mathrm{ml}$ y la alcalinización de la orina para alcanzar un $\mathrm{pH}$ de alrededor de 7,5 son las bases del tratamiento de la cistinuria durante el embarazo. Los fármacos como la penicilamina y la alfa-mercaptopropionil glicina, que separan los enlaces cistina-cistina mediante el intercambio de enlaces disulfuro con residuos de cisteína, están contraindicados en el embarazo debido a los posibles efectos adversos fetales demostrados en ratas ${ }^{6}$.

\section{Manejo quirúrgico}

Si el tratamiento conservador falla, y en casos de dolor incontrolable, empeoramiento del cuadro clínico, vómitos persistentes, fiebre, sepsis, complicaciones obstétricas, riñón solitario, obstrucción ureteral bilateral y cálculos ureterales mayores de $1 \mathrm{~cm}$, se recomienda tratamiento quirúrgico $0^{6,20,59}$.

\section{DRENAJE RENAL}

Hoy por hoy, el drenaje renal solo está indicado cuando no se aconseja o no se dispone de un tratamiento quirúrgico definitivo ${ }^{6}$. El abordaje urológico para el drenaje renal en el embarazo incluye el stent ureteral y la nefrostomía percutánea ${ }^{20}$. El drenaje solo puede estar indicado en pacientes con cálculos con infección activa, cálculos grandes o bilaterales, malformaciones anatómicas o complicaciones obstétricas ${ }^{6}$.

Se ha demostrado que tanto los stents ureterales como el catéter de nefrostomía son igualmente eficaces para drenar un sistema colector infectado, y la instauración de uno u otro dependerá de la particularidad de cada caso, la disponibilidad de recursos y las preferencias del cirujano y de la paciente ${ }^{6,17}$. Ambos presentan ventajas y desventajas. La ventaja que tienen los stents ureterales sobre sobre los catéteres de nefrostomía es que brindan mayor comodidad a la paciente por no tener material exteriorizado, y su desventaja es que pueden causar molestias suprapúbicas, irritación y dolor ${ }^{10,60}$. Por otro lado, la ventaja de la nefrostomía percutánea es que resulta más asequible y tiene éxito en el $90 \%$ de las pacientes; su desventaja es que se asocia a molestias en el costado, hemorragias, desplazamiento del catéter e infecciones ${ }^{10,20,61}$. Ambos métodos son un procedimiento temporal. A causa de los cambios metabólicos que ocurren en el embarazo, cualquiera de los dos dispositivos puede infectarse, desprenderse, bloquearse 0 incrustarse, y esto hace necesario su cambio cada 4-6 semanas. De igual manera, cabe señalar que estos procedimientos son mínimamente invasivos y se pueden realizar con anestesia mínima (local o general) bajo guía ecográfica; sin embargo, la inserción del stent ureteral a menudo se realiza bajo guía fluoroscópica, lo cual no se recomienda en el primer trimestre de embarazo ${ }^{17,61}$. En este sentido, se debe enfatizar en recomendar su inserción bajo guía ecográfica, sobre todo en este periodo de la gestación.

Drescher, et al. ${ }^{62}$ identificaron el riesgo de presentar infección del tracto urinario y parto pretérmino teniendo en cuenta los distintos manejos de la nefrolitiasis en la gestante, y concluyeron que la nefrostomía percutánea representó un mayor riesgo de infección del tracto urinario y de parto pretérmino (odds ratio [OR]: 2,3), a diferencia la colocación de stent ureteral $(\mathrm{OR}: 1,5)$ y del manejo conservador (OR: 1,3), que mostraron un menor riesgo para la génesis de estas complicaciones.

\section{TRATAMIENTO QUIRÚRGICO DEFINITIVO DE LOS CÁLCULOS}

Para las mujeres embarazadas sin signos de infección, complicaciones obstétricas, escenarios intrincados de cálculos o cuyo embarazo ha pasado el primer trimestre, el tratamiento quirúrgico definitivo es una opción viable ${ }^{6}$.

Los avances tecnológicos han permitido la implementación de nuevas técnicas, como la ureteroscopia (URS), para el abordaje terapéutico de la litiasis renal, disminuyendo así la práctica de la cirugía abierta ${ }^{48,63}$. La URS es una técnica segura durante el embarazo, permite una mejor visualización del cálculo mediante la utilización de un instrumento rígido, semirrígido o flexible ${ }^{20}$, se puede realizar bajo anestesia general, espinal o local, con monitorización fetal durante y después del procedimiento, y además brinda beneficios a la paciente, como menor estancia hospitalaria y menor tiempo de recuperación ${ }^{15,63,64}$. Se ha convertido en una alternativa aceptada para pacientes que requerirían múltiples cambios de catéter y aquellas que no pueden tolerar un stent ureteral o un catéter de nefrostomía debido a la presencia de molestias. La URS se puede realizar con una emisión limitada de rayos $\mathrm{X}$, utilizando un escudo protector en la pelvis de la paciente, o solo 


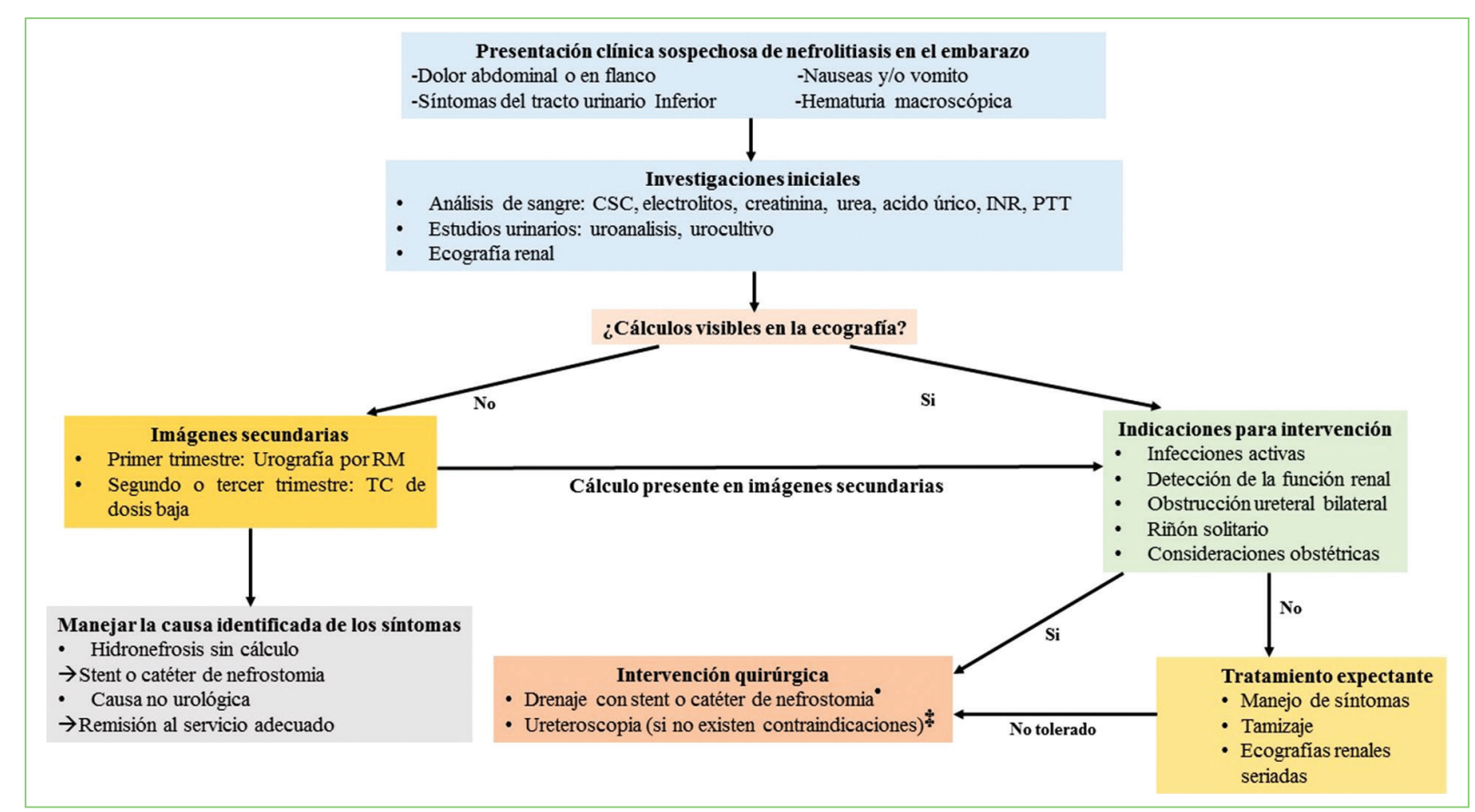

Figura 2. Algoritmo para el abordaje diagnóstico y terapéutico ante la sospecha de nefrolitiasis en una paciente embarazada. CSC: conteo sanguíneo completo; INR: International Normalized Ratio; RM: resonancia magnética; PTT: tiempo parcial de tromboplastina; TC: tomografía computarizada. (Adaptado de Bjazevic y Razvi ${ }^{17}$.)

*Se debe cambiar el catéter cada 4-6 semanas.

†Contraindicaciones: infección, cálculos múltiples, anatomía anormal, consideraciones obstétricas.

con guía ecográfica completa ${ }^{6}$. Entre las complicaciones que se pueden presentar en la paciente embarazada se encuentran el trabajo de parto prematuro, pero con un riesgo muy bajo (0-1\%), lo cual hace confiable su uso ${ }^{65}$. La URS está contraindicada en algunas situaciones, como cálculos múltiples, malformaciones anatómicas renales, complicaciones obstétricas e infección activa. A pesar de que la URS no está contraindicada en el primer trimestre de embarazo, si el procedimiento no es de urgencia es preferible realizarlo en el segundo trimestre $^{66}$ debido a los posibles riesgos que puede generar la anestesia en el feto en el primer trimes$\operatorname{tre}^{17,67}$. Durante las primeras 12 semanas de gestación, el feto es más vulnerable a los efectos teratogénicos. Además, la tasa de aborto baja al $5,6 \%$ en el segundo trimestre del embarazo, en comparación con el 12-15\% durante el primero, y la incidencia de parto prematuro es menor en el segundo trimestre que en el tercero. En teoría, el riesgo de teratogénesis es más bajo a partir del segundo trimestre ${ }^{68}$. En este sentido, es ideal postergar el uso de anestésicos para el segundo trimestre de gestación.

Los tratamientos alternativos, como la litotricia por ondas de choque y la nefrolitotomía percutánea, están contraindicados durante el embarazo por tener efectos teratogénicos ${ }^{42}$; si se requiere utilizar una de estas técnicas, es conveniente esperar al periodo posparto ${ }^{36}$.

La incidencia de nefrolitiasis en las mujeres ha aumentado en las últimas décadas, y por tanto se espera que durante el embarazo también aumente ${ }^{17}$. El cólico renal agudo en el embarazo es una situación clínica difícil que tiene posibles complicaciones graves para la madre y el feto, y requiere un enfoque de manejo reflexivo y multidisciplinario. El algoritmo para el abordaje diagnóstico y terapéutico de esta revisión se presenta en la figura 2.

\section{Conclusiones}

La paciente gestante con nefrolitiasis es un desafío clínico para el personal médico, debido a que puede confundirse con otras patologías agudas que comparten la misma presentación clínica. Además, la dificultad aumenta por la limitación en las pruebas diagnósticas que se pueden utilizar en la gestante sin poner en riesgo al feto. Actualmente, la litiasis renal en la embarazada es una patología que está siendo infradiagnosticada e ignorada, y por esta razón se hace hincapié en la 
importancia de la sospecha clínica con el fin de instaurar un diagnóstico precoz y un tratamiento oportuno, y así evitar las complicaciones que puedan derivarse. Por ello, el médico debe tener el conocimiento y las destrezas para brindar un abordaje adecuado de dicha patología de acuerdo con el escenario en que se presente.

\section{Financiación}

Los autores declaran que no han recibido ningún financiamiento para escribir el presente artículo.

\section{Conflicto de intereses}

Los autores declaran que no tienen ningún conflicto de intereses relacionado con el contenido del presente artículo.

\section{Responsabilidades éticas}

Protección de personas y animales. Los autores declaran que para esta investigación no se han realizado experimentos en seres humanos ni en animales.

Confidencialidad de los datos. Los autores declaran que en este artículo no aparecen datos de pacientes.

Derecho a la privacidad y consentimiento informado. Los autores declaran que en este artículo no aparecen datos de pacientes.

\section{Bibliografía}

1. Sánchez A. Cálculos renales: ¿es posible su prevención? Rev Med Rosario. 2018;84:7-8.

2. Guillén R, Funes $P$, Echagüe $G$. Morphologic analysis of voluminous and staghorn kidney stones. Mem Inst Invest Cienc Salud. 2016;14:61-7.

3. Masselli G, Derme M, Bernieri M, Polettini E, Casciani E, Monti R, et al. Stone disease in pregnancy: imaging-guided therapy. Insights Imaging. 2014;5:691-6.

4. Rosenberg E, Sergienko R, Abu-Ghanem S, Wiznitzer A, Romanowsky I, Neulander, et al. Nephrolithiasis during pregnancy: characteristics, complications, and pregnancy outcome. World J Urol. 2011;29:743-7.

5. Yatzidis H. Gestational urinary hyperthiosulfaturia protects hypercalciuric normal pregnant women from nephrolithiasis. Int Urol Nephrol. 2004;36:445-9.

6. Pedro R, Das K, Buchholz N. Urolithiasis in pregnancy. Int J Surg. 2016;36:688-92.

7. Masselli G, Derme M, Laghi F, Polettini E, Brunelli R, Framarino M, et al Imaging of stone disease in pregnancy. Abdom Imaging. 2013;38:1409-14

8. Cox S, Reid F. Urogynaecological complications in pregnancy: an overview. Obstet Gynaecol Reprod Med. 2018;28:78-82.

9. Masselli G, Weston M, Spencer J. The role of imaging in the diagnosis and management of renal stone disease in pregnancy. Clin Radiol. 2015;70:1462-71.

10. Genin A, Meria P, Daudon M, Desgrandchamps F. Litiasis urinaria $y$ embarazo. EMC Urol. 2012;44:1-11.

11. Martínez P, Colorado S, Del Barco L. Urolitiasis y embarazo. Ginecol Obstet Mex. 2007;75:357-63.

12. García Nieto VM, Luis Yanes MI. Capítulo 7: Litiasis renal. En: Nefrología al día. p. 133-47. Disponible en: https://revistanefrologia.com/index. php?p=revista and tipo=pdf-simple and pii=XX342164212000524

13. Corbo J, Wang J. Kidney and ureteral stones. Emerg Med Clin North Am. 2019;37:637-48.

14. Pfau A, Knauf F. Update on nephrolithiasis: core curriculum 2016. Am J Kidney Dis. 2016;68:973-85.
15. Hofmeyr GJ, Lawrie TA, Atallah AN, Duley L, Torloni MR. Calcium supplementation during pregnancy for preventing hypertensive disorders and related problems. Cochrane Database Syst Rev. 2018;(10):CD001059.

16. Imdad A, Bhutta ZA. Effect of calcium supplementation during pregnancy on maternal, fetal and birth outcomes. Paediatr Perinat Epidemiol. 2012;26 (Suppl 1):138-52.

17. Bjazevic J, Razvi H. Stones in pregnancy and pediatrics. Asian J Urol. 2018:5:223-34.

18. Ferraro P, Robertson W, Unwin R. Renal stone disease. Medicine Obstruction and Infection (United Kingdom). 2019;47:537-40.

19. Kazma J, van den Anker J, Allegaert K, Dallmann A, Ahmadzia H. Anatomical and physiological alterations of pregnancy. J Pharmacokinet Pharmacodyn. 2020;47:271-85.

20. Charalambous S, Fotas A, Rizk D. Urolithiasis in pregnancy. Int Urogynecol J Pelvic Floor Dysfunct. 2009;20:1133-6.

21. Al-Mamari S. Urolithiasis in clinical practice: special conditions in urinary lithiasis. Springer Cham; 2017. p. 19-25.

22. Navalón-Verdejo P, Sánchez-Ballester F, Pallas-Costa Y, Cánovas-Ivorra J, Ordoño-Domínguez $\mathrm{F}$, Juan-Escudero J, et al. Acerca de la hidronefrosis sintomática en la gestante. Arch Esp Urol. 2005;58:977-82.

23. Reinstatler L, Khaleel S, Pais V. Association of pregnancy with stone formation among women in the United States: a NHANES analysis 2007 to 2012. J Urol. 2017;198:389-93.

24. Korkes F, Costa-Rauen E, Pfeferman-Heilberg I. Urolithiasis and pregnancy. J Bras Nefrol. 2014;36:389-95.

25. Bacallao R, Mañalich R, Gutiérrez F, Badell A. Composición de las urolitiasis en pacientes cubanos por sexo. Rev Cub Invest Biomed. 2015;34:328-36.

26. Tan Y, Cha D, Gupta M. Management of stones in abnormal situations. Urol Clin North Am. 2013;40:79-97.

27. Parulkar BG, Hopkins TB, Wollin MR, Howard PJ, Lal A. Renal colic during pregnancy: a case for conservative treatment. J Urol. 1998;159:365-8.

28. Spivacow F, Del Valle E, Lores E, Rey P. Kidney stones: composition, frequency and relation to metabolic diagnosis. Medicina (Buenos Aires). 2016;76:343-8.

29. Ross A, Handa S, Lingeman J, Matlaga B. Kidney stones during pregnancy: an investigation into stone composition. Urol Res. 2008;36:99-102.

30. Courbebaisse M, Daudon M. Litiasis renal y nefrocalcinosis. EMC - Tratado de Medicina. 2016;20:1-6.

31. Mayans L. Nephrolithiasis. Prim Care Clin Office Pract. 2019;46:203-12.

32. Kobayashi T, Nishizawa K, Mitsumori K, Ogura K. Impact of date of onset on the absence of hematuria in patients with acute renal colic. J Urol. 2003;170 (4 pt 1):1093-6.

33. Burgess KL, Gettman MT, Rangel LJ, Krambeck AE. Diagnosis of urolithiasis and rate of spontaneous passage during pregnancy. J Urol. 2011;186:2280-4.

34. Clennon E, Duty B, Caughey A. 784: Obstetric outcomes of pregnancy complicated by urolithiasis: a retrospective cohort study. Am J Obstet Gynecol. 2018;218:S468.

35. Tublin M, Levine D, Thurston W, Wilson S. The kidney and urinary tract. En: Rumack C, Levine D, editores. Diagnostic ultrasound. $5^{\text {th }}$ ed. Philadelphia: Elsevier; 2019. p. 310-80.

36. Vallone G, Napolitano G, Fonio P, Antinolfi G, Romeo A, Macarini L, et al. US detection of renal and ureteral calculi in patients with suspected renal colic. Crit Ultrasound J. 2013;5 (Suppl 1):S3.

37. Eisner B, Eisenberg ML, Stoller ML. Influence of body mass index on quantitative 24-hour urine chemistry studies in children with nephrolithiasis. J Urol. 2009;182:1142-5.

38. American College of Obstetricians and Gynecologists. Committee Opinion No. 723: Guidelines for diagnostic imaging during pregnancy and lactation. Obstet Gynecol. 2017;130(4):e210-6.

39. Arenas J, Baldwin D. Techniques for minimizing radiation exposure during evaluation, surgical treatment, and follow-up of urinary lithiasis. Curr Urol Rep. 2015;16:45.

40. Semins MJ, Feng Z, Trock B, Bohlman M, Hosek W, Matlaga BR, et al. Evaluation of acute renal colic: a comparison of non-contrast CT versus 3-T non-contrast HASTE MRI urography. Urolithiasis. 2013;41:43-6.

41. Spencer JA, Chahal R, Kelly A, Taylor K, Eardley I, Lloyd SN. Evaluation of painful hydronephrosis in pregnancy: magnetic resonance urographic patterns in physiological dilatation versus calculous obstruction. J Urol. 2004;171:256-60.

42. Semins JM, Matlaga BR. Management of urolithiasis in pregnancy. Int $\mathrm{J}$ Womens Health. 2013;5:599-604.

43. McCollough $\mathrm{CH}$, Schueler BA, Atwell TD, Braun NN, Regner DM, Brown DL, et al. Radiation exposure and pregnancy: when should we be concerned? Radiographics. 2007;27:909-17.

44. White WM, Zite NB, Gash J, Waters WB, Thompson W, Klain FA Low-dose computed tomography for the evaluation of flank pain in the pregnant population. J Endourol. 2007;21:1255-60.

45. White WM, Johnson EB, Zite NB, Beddies J, Krambeck AE, Hyams E, et al. Predictive value of current imaging modalities for the detection of urolithiasis during pregnancy: a multi-center, longitudinal study. J Urol. 2013;189: 931-4. 
46. Fulgham PF, Assimos DG, Pearle MS, Preminger GM. Clinical effectiveness protocols for imaging in the management of ureteral calculous disease: AUA technology assessment. J Urol. 2013;189:1203-13.

47. Semins MJ, Matlaga BR. Medical evaluation and management of urolithiasis. Ther Adv Urol. 2010;2:3-9.

48. Ordon M, Dirk J, Slater J, Kroft J, Dixon S, Welk B. Incidence, treatment and implications of kidney stones during pregnancy: a matched population-based cohort study. J Endourol. 2020;34:215-21.

49. Dion M, Violette $\mathrm{P}$, Razvi H. Managing stones in pregnancy: an update. Clin Pract. 2014;11:699-710.

50. Semins M, Matlaga B. Kidney stones and pregnancy. Adv Chronic Kidney Dis. 2013;20:260-4

51. Gao X, Fang Z, Lu C, Shen R, Dong H, Sun Y. Management of staghorn stones in special situations. Asian J Urol. 2020;7:130-8.

52. Castro-Garcés L. Analgesia en la paciente obstétrica. Rev Mex Anest 2019;42:194-7.

53. Sosa L. Farmacoterapia durante el embarazo. Arch Med Intern. 2009;31 87-92.

54. Dobiesz VA, Robinson D. Drug Therapy in Pregnancy. En: Rosen's Emergency medicine concepts and clinical practice. $9^{\text {th }}$ ed. Philadelphia: Elsevier; 2018. p. 2277-95

55. Theriault B, Morin F, Cloutier J. Safety and efficacy of tamsulosin as medical expulsive therapy in pregnancy. World J Urol. 2020;38:2301-6.

56. Woolley B. Towards evidence based emergency medicine: best BETs from the Manchester Royal Infirmary. Use of tamsulosin in patients with urinary calculi to increase spontaneous stone passage. Emerg Med J. 2007;24: 725-6

57. Sandoval $P$, Sandoval $C$. Uso de fármacos durante el embarazo. Horiz Med. 2018;18:71-9.
58. Aggarwal S, Brown M. Renal physiology and complications in normal pregnancy. En: Feehally J, Floege J, Tonelli M, Johnson RJ, editores. Comprehensive clinical nephrology. $6^{\text {th }}$ ed. Philadelphia: Elsevier; 2019. p. 502-21.

59. Srirangam SJ, Hickerton B, Van Cleynenbreugel B. Management of urinary calculi in pregnancy: a review. J Endourol. 2008;22:867-75.

60. Ishii H, Aboumarzouk O, Somani B. Current status of ureteroscopy for stone disease in pregnancy. Urolithiasis. 2014;42:1-7.

61. Núñez M, Chiconi M, Linares G, Aveni F, Pilot M, Albino G. Litiasis renal: ¿puede el abordaje percutáneo resolver todos los casos? Rev Arg Urol. 2018;83:132-7.

62. Drescher M, Blackwell R, Patel P, Kuo P, Turk T, Baldea, K. Antepartum nephrolithiasis and the risk of preterm delivery. Urolithiasis. 2019;47:441-8.

63. Pineda J, Martínez G, Marín D, Viveros C, Torres J, Pineda E. Tratamiento contemporáneo de la litiasis renal piélica: a propósito de un caso. Rev Fac Med Mex. 2018;61:16-21.

64. Granados E, Caparrós J, Rousaud A. Treatment of lithiasis during pregnancy. Arch Esp Urol. 1991:44:979-82.

65. Liu G, Wang J, Li J, Zheng J, Huang Z, Ye Z. Urolithiasis in pregnancy: survey in clinical epidemiology. J Huazhong Univ Sci Technol. 2011;31: 226-30.

66. Tekgül S, Dogan HS, Hoebeke P, Kočvara R, Nijman JM, Radmayr C, et al. EAU Guidelines on Urolithiasis. European Association of Urology; 2021. Disponible en: https://uroweb.org/wp-content/uploads/EAU-Guidelines-on-Urolithiasis-2021 pdf

67. Birnbach DJ, Bateman BT. Obstetric anesthesia: leading the way in patient safety. Obstet Gynecol Clin North Am. 2019;46:329-37.

68. Socha-García NI, Gómez-Morant JC, Holguín-González E. Cirugía no obstétrica durante el embarazo. Rev Col Anest. 2011;39:360-73. 